\title{
Searching for dark matter with neutron star mergers and quiet kilonovae
}

\author{
Joseph Bramante, ${ }^{1,2}$ Tim Linden, ${ }^{3}$ and Yu-Dai Tsai ${ }^{2,4}$ \\ ${ }^{1}$ CPARC and Department of Physics, Engineering Physics, and Astronomy, \\ Queen's University, Kingston, Ontario, Canada K7L 2S8 \\ ${ }^{2}$ Perimeter Institute for Theoretical Physics, Waterloo, Ontario, Canada N2L $2 Y 5$ \\ ${ }^{3}$ CCAPP and Department of Physics, The Ohio State University, Columbus 43210, Ohio, USA \\ ${ }^{4}$ Laboratory for Elementary Particle Physics, Cornell University, Ithaca 14853, New York, USA
}

(Received 8 June 2017; revised manuscript received 4 February 2018; published 12 March 2018)

\begin{abstract}
We identify new astrophysical signatures of dark matter that implodes neutron stars (NSs), which could decisively test whether NS-imploding dark matter is responsible for missing pulsars in the Milky Way galactic center, the source of some $r$-process elements, and the origin of fast-radio bursts. First, NSimploding dark matter forms $\sim 10^{-10}$ solar mass or smaller black holes inside neutron stars, which proceed to convert neutron stars into 1.5 solar mass black holes (BHs). This decreases the number of neutron star mergers seen by LIGO/Virgo (LV) and associated merger kilonovae seen by telescopes like DES, BlackGEM, and ZTF, instead producing a population of "black mergers" containing $~ 1.5$ solar mass black holes. Second, dark matter-induced neutron star implosions may create a new kind of kilonovae that lacks a detectable, accompanying gravitational signal, which we call "quiet kilonovae." Using DES data and the Milky Way's r-process abundance, we constrain quiet kilonovae. Third, the spatial distribution of neutron star merger kilonovae and quiet kilonovae in galaxies can be used to detect dark matter. NS-imploding dark matter destroys most neutron stars at the centers of disc galaxies, so that neutron star merger kilonovae would appear mostly in a donut at large radii. We find that as few as ten neutron star merger kilonova events, located to $\sim 1 \mathrm{kpc}$ precision could validate or exclude dark matter-induced neutron star implosions at $2 \sigma$ confidence, exploring dark matter-nucleon cross-sections 4-10 orders of magnitude below current direct detection experimental limits. Similarly, NS-imploding dark matter as the source of fast radio bursts can be tested at $2 \sigma$ confidence once 20 bursts are located in host galaxies by radio arrays like CHIME and HIRAX.
\end{abstract}

DOI: 10.1103/PhysRevD.97.055016

\section{INTRODUCTION}

Uncovering the identity and interactions of dark matter would deepen our understanding of fundamental physics and the origin of our universe. Because dark matter is abundant in galactic halos, it may be revealed through its impact on stars. Indeed, a number of ongoing astrophysical searches may be unveiling dark matter that forms black holes inside and implodes old neutron stars [1-5], thereby creating r-process elements [6], and possibly fast radio bursts [7].

Traditional neutron star-based searches for asymmetric dark matter have relied on old pulsars in the Milky Way, whose old age bounds the capture rate of asymmetric dark matter, which can eventually form black holes inside of neutron stars. Finding old pulsars in the Milky Way's galactic center, where dark matter is denser, more dark matter is

Published by the American Physical Society under the terms of the Creative Commons Attribution 4.0 International license. Further distribution of this work must maintain attribution to the author(s) and the published article's title, journal citation, and DOI. Funded by SCOAP. captured, and therefore pulsars potentially implode faster, would advance the frontier of asymmetric dark matter detection. However, extensive radio surveys of the Milky Way galactic center have not found enough pulsars to conclusively strengthen or invalidate the hypothesis that dark matter implodes neutron stars in that region. Fortuitously, the unprecedented sensitivity of laser interferometer gravitational wave detectors at LIGO/Virgo [8], the broad optical purview of DES [9,10], BlackGEM [11], and other optical telescopes, as well as kilo-channel radio reception at CHIME [12] and HIRAX [13], are better scrutinizing the dynamics of neutron stars and black holes. Indeed, recently a neutron star merger was found both in gravitational waves [14] and follow-on telescope observations [15].

This article demonstrates how the combined statistics of neutron star mergers observed in galaxies can be used to unmask dark matter with nucleon scattering cross-sections orders of magnitude smaller than the present reach obtained from neutron stars in the Milky Way, and up to ten orders of magnitude beyond any planned underground dark matter detection experiment. This new search method applies to many asymmetric dark matter models [16,17], including 
$\mathrm{keV}-\mathrm{PeV}$ mass bosons [1-5,18-24], keV-EeV mass selfinteracting fermions $[2,25,26]$, and $\gtrsim \mathrm{PeV}$ mass bosons and fermions $[4,6,27,28]$.

Additionally, this article details distinct signatures of dark matter-induced neutron star implosions, which can be discovered by upcoming gravitational, optical, and radio surveys. NS-imploding dark matter may produce detectable quiet kilonovae: a kilonova powered by the ejection of neutron star fluid during the dark matter-induced implosion of a neutron star. These quiet kilonovae would be "quiet" and distinct from the now-observed neutron star merger kilonovae, in that they would not produce gravitational waves detectable by LIGO/Virgo. The remainder of this document is structured as follows: in Sec. II we review asymmetric dark matter that implodes neutron stars and introduce the normalized implosion time. Section III details how neutron star populations in disc galaxies are altered by NS-imploding dark matter. Section IV details prospects for finding primordial black holes (PBHs) using neutron star mergers and quiet kilonova. Section $V$ provides the first constraint on "quiet kilonovae." Section VI details a major result of this work: that NS mergers can be used as an incisive new search for dark matter. A location and rate analysis testing whether fast radio bursts originate from dark matter-induced neutron star implosions is presented in Sec. VII. In Sec. VIII, we conclude. Appendix A presents the cumulative distributions functions employed in Secs. VII and VI and Appendix B provides details of neutron star implosions induced by heavy asymmetric dark matter.

\section{DARK MATTER-INDUCED NEUTRON STAR IMPLOSIONS}

Once enough dark matter has accumulated in a neutron star's interior, dark matter may collapse into a small ( $\left.\lesssim 10^{-10} \mathrm{M}_{\odot}\right)$ black hole that subsequently consumes the neutron star $[1,3,6]$. We begin by defining a useful variable combination, the "normalized implosion time," which relates dark matter-induced NS implosions occurring at different radii from a galactic center, where the local dark matter density $\rho_{\mathrm{x}}$ and velocity dispersion $v_{\mathrm{x}}$ will be different, see Fig. 1. The maximum mass accumulation rate of dark matter into a NS is [29]

$$
\begin{aligned}
\dot{m}_{\mathrm{x}} & =\pi \rho_{\mathrm{x}} \frac{2 G M R}{v_{\mathrm{x}}}\left(1-\frac{2 G M}{R}\right)^{-1} \\
& \simeq \frac{10^{26} \mathrm{GeV}}{s}\left(\frac{\rho_{\mathrm{x}}}{\mathrm{GeV} / \mathrm{cm}^{3}}\right)\left(\frac{200 \mathrm{~km} / \mathrm{s}}{v_{\mathrm{x}}}\right),
\end{aligned}
$$

where $M$ and $R$ are the mass and radius of the neutron star and $G$ is Newton's constant. The time until NS implosion scales inversely with the mass accumulation rate, $t_{\mathrm{c}} \propto \dot{m}_{\mathrm{x}}^{-1}$; therefore $t_{\mathrm{c}}$ is proportional to the dark matter velocity dispersion divided by density, $t_{\mathrm{c}} \propto v_{\mathrm{x}} / \rho_{\mathrm{x}}$. Furthermore, $v_{\mathrm{x}}$ and $\rho_{x}$ are the only quantities in $\dot{m}_{\mathrm{x}}$ that depend on the
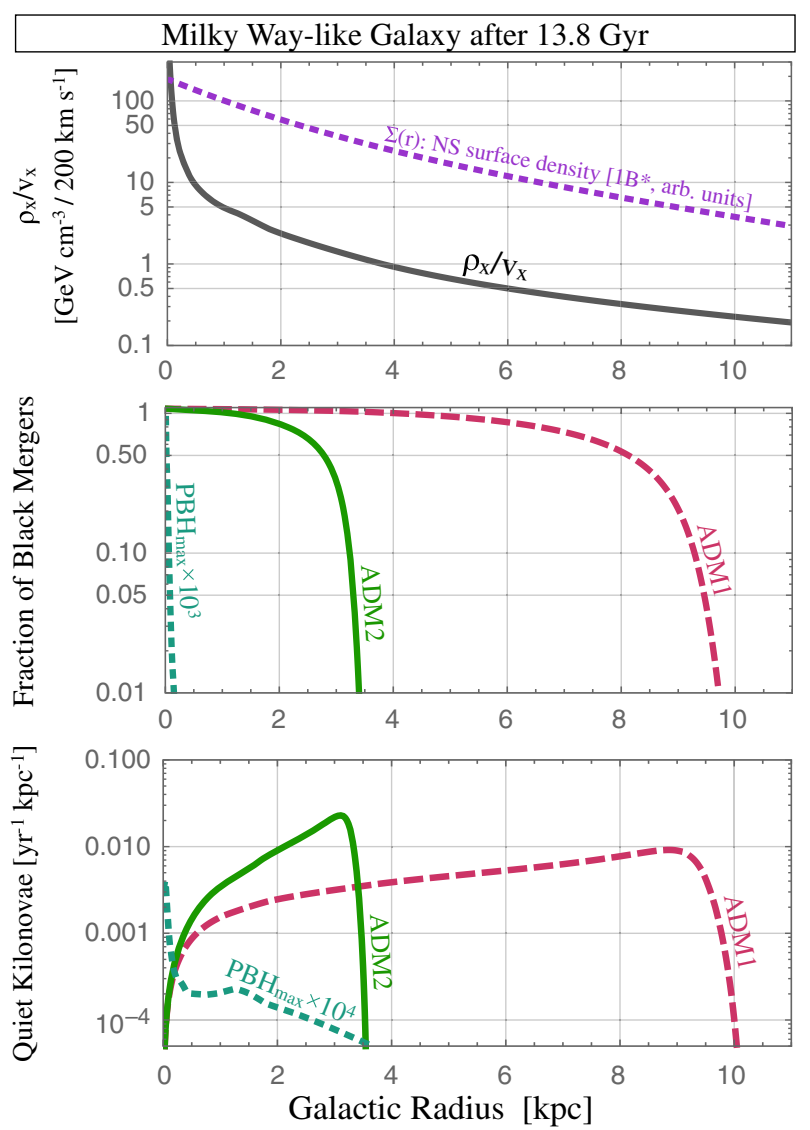

FIG. 1. The dark matter density over velocity $\rho_{\mathrm{x}} / v_{\mathrm{x}}$ and NS surface density $\Sigma$ in an MWEG (top), the total fraction of imploded neutron stars (middle), and the rate for quiet kilonovae, aka dark matter-induced NS implosions, per yr per $\mathrm{kpc}$ (bottom), are given as a function of distance from the center of a Milky Way-like galaxy for two asymmetric dark matter models $\mathrm{ADM} 1$ and $\mathrm{ADM} 2$, defined by $t_{\mathrm{c}} \rho_{\mathrm{x}} / v_{\mathrm{x}}=3$ and $15 \mathrm{Gyr} \mathrm{GeV} / \mathrm{cm}^{3}(200 \mathrm{~km} / \mathrm{s})^{-1} \cdot \mathrm{PBH}_{\max }$ is a maximally NSimploding model described in Appendix B. For visibility, the PBH curve has been augmented by 3 and 4 orders of magnitude.

galactocentric radius $r$. It follows that for dark matter which implodes NSs in time $t_{\mathrm{c}}$, the quantity

$$
t_{\mathrm{c}} \frac{\rho_{\mathrm{x}}}{v_{\mathrm{x}}}=\text { Constant } \times\left[\mathrm{Gyr} \frac{\mathrm{GeV} / \mathrm{cm}^{3}}{200 \mathrm{~km} / \mathrm{s}}\right]
$$

which we call the normalized implosion time, ${ }^{1}$ is independent of $r$. Throughout we will normalize $t_{\mathrm{c}} \rho_{\mathrm{x}} / v_{\mathrm{x}}$ to a typical dark matter density $\left(\mathrm{GeV} / \mathrm{cm}^{3}\right)$ and velocity dispersion $(200 \mathrm{~km} / \mathrm{s})$ for a disc galaxy.

The value of $t_{\mathrm{c}} \rho_{\mathrm{x}} / v_{\mathrm{x}}$ for a specific dark matter model can be determined by calculating the time for dark matter with

\footnotetext{
${ }^{1}$ The units given in square brackets in Eq. (2) might be read as "A neutron star will implode in one gigayear for local dark matter density $\mathrm{GeV} / \mathrm{cm}^{3}$ and dark matter velocity dispersion $200 \mathrm{~km} / \mathrm{s} . "$
} 
local density $\rho_{\mathrm{x}}$ and relative velocity $v_{\mathrm{x}}$ to implode a neutron star. While many asymmetric dark matter models implode neutron stars [1-6,18-20,25-27], we will focus on heavy $m_{\mathrm{x}} \gtrsim \mathrm{PeV}$ asymmetric dark matter as a simple example. In the case of heavy asymmetric dark matter, the critical mass of dark matter required to form a small black hole is $M_{\mathrm{crit}}^{\mathrm{f}} \sim \frac{m_{\mathrm{pl}}^{3}}{m_{\mathrm{x}}^{2}}$ for dark fermions with mass $m_{\mathrm{x}}$ [27], and $M_{\text {crit }}^{\mathrm{b}} \sim 0.12 \sqrt{\lambda} \frac{m_{\mathrm{pl}}^{3}}{m_{\mathrm{x}}^{2}}$ for dark scalars with selfinteraction potential $V(\phi)=\lambda|\phi|^{4}$ [30]. In these models, the neutron star will implode shortly after it collects a critical mass of dark matter at time $t_{\mathrm{c}} \simeq M_{\text {crit }} / \dot{m}_{\mathrm{x}}$, where this expression assumes all dark matter passing through the neutron star is captured-see Appendix B for details and for the scaling of dark matter-nucleon cross section with $t_{\mathrm{c}} \rho_{\mathrm{x}} / v_{\mathrm{x}}$. Then the value of the galactic radial invariant $t_{\mathrm{c}} \rho_{\mathrm{x}} / v_{\mathrm{x}}$ is

$$
\begin{aligned}
& \left.t_{\mathrm{c}} \frac{\rho_{\mathrm{x}}}{v_{\mathrm{x}}}\right|_{\mathrm{f}}=\left(\frac{10 \mathrm{PeV}}{m_{\mathrm{x}}}\right)^{2} 15 \mathrm{Gyr} \frac{\mathrm{GeV} / \mathrm{cm}^{3}}{200 \mathrm{~km} / \mathrm{s}} \\
& \left.t_{\mathrm{c}} \frac{\rho_{\mathrm{x}}}{v_{\mathrm{x}}}\right|_{\mathrm{b}}=\left(\frac{\lambda}{1}\right)^{1 / 2}\left(\frac{3 \mathrm{PeV}}{m_{\mathrm{x}}}\right)^{2} 20 \mathrm{Gyr} \frac{\mathrm{GeV} / \mathrm{cm}^{3}}{200 \mathrm{~km} / \mathrm{s}},
\end{aligned}
$$

for heavy asymmetric fermions and bosons, respectively.

\section{BLACK MERGERS, QUIET KILONOVAE, AND R-PROCESS DONUTS}

NS-imploding dark matter creates an unexpected population of low mass $\sim 1.5 \mathrm{M}_{\odot}$ black holes (BHs), depleting the expected population of NSs. This in turn would alter the number of merging neutron stars that would be seen by LIGO/Virgo, along with their accompanying merger kilonovae, which are the days-long luminous outbursts from beta decaying neutrons ejected when NSs fall into a BH or another NS [31,32].

We now determine the number and position of neutron stars converted to BHs by dark matter in a Milky Waylike galaxy. With some subtleties that we will address, our findings for a typical $13 \mathrm{Gyr}$ old $\sim 10^{12} \mathrm{M}_{\odot}$ disc galaxy can be applied to events in different galaxies, using a Milky Way equivalent galaxy (MWEG) volumetric conversion for merger and kilonova rates, i.e., one MWEG per $(4.4 \mathrm{Mpc})^{3}$ [33].

The number of neutron stars converted into BHs by dark matter in an MWEG will depend on the historic neutron star formation rate in the galaxy, the dynamics and final positions of neutron stars after formation, the dark matter halo density profile, and the relative velocity of dark matter with respect to neutron stars. We model the historic star formation rate $\dot{M}^{*}(t)$ using a global fit to astronomical data ([34], Table 1, Column 2). While we use $\dot{M}^{*}(t)$ to determine the relative historic rate of neutron star formation, we normalize the total rate to $10^{9}$ neutron star births over the MWEG lifetime [3537]. At birth, it has been observed that neutron stars receive natal kicks which result in an initial velocity boost of $\sim 250 \mathrm{~km} / \mathrm{s}[38,39]$. A recent study of neutron star dynamics in an MWEG has found that most ( $\gtrsim 80 \%)$ neutron stars are retained within a $\sim$ kiloparsec of the MWEG disc plane, with a NS surface density $\Sigma(r)$. We therefore model the MWEG neutron star distribution as a thin disk with surface density $\Sigma(r)$ given by models $1 \mathrm{~B}^{*}$ (and $1 \mathrm{C}^{*}$ as indicated for comparison) in [37].

To model dark matter in an MWEG, we use an NFW dark matter halo density profile [40], $\rho_{\mathrm{x}}(r)=$ $\rho_{0}\left(r / R_{\mathrm{S}}\right)^{-1}\left(1+r / R_{\mathrm{S}}\right)^{-2}$, with dark matter density normalization $\rho_{0}=0.3 \mathrm{GeV} / \mathrm{cm}^{3}$ and scale factor $R_{\mathrm{S}}=20 \mathrm{kpc}$. To approximate the dark matter velocity dispersion in an MWEG, we match the phenomenological fit of Sofue to stellar velocities in the Milky Way ([41], Fig. 11). With the star formation rate, neutron star distribution and dark matter properties specified, the fraction of neutron stars at radius $r$ converted to solar mass BHs is given by $F_{\mathrm{BH}}(r)=\frac{\int_{0}^{\operatorname{Max}\left[t_{\mathrm{u}}-t_{\mathrm{c}}(r), 0\right]} \dot{M}^{*}(t) d t}{\int_{0}^{t_{\mathrm{u}}} \dot{M}^{*}(t) d t}$, where $t_{\mathrm{u}} \sim 13.8 \mathrm{Gyr}$ is the lifetime of the universe and $t_{\mathrm{c}}(r)$ is the collapse time at radius $r$, obtainable from Eq. (3). Similarly, the rate of neutron star implosions (and also quiet kilonovae) per unit galactocentric radius is given by $R_{\mathrm{qk}}=$ $2 \pi r \Sigma(r) \dot{M}^{*}\left(t_{\mathrm{u}}-t_{\mathrm{c}}(r)\right)$. In Fig. 1, we plot the fraction of neutron stars converted to $\mathrm{BHs}$ along with the rate of neutron star implosions per year per kpc, both as a function of galactocentric radius, for a 13 Gyr old MWEG. In Table I, we show how standard rates for compact object mergers would be altered, and display dark matter-induced neutron star implosion rates, for a few values of $t_{\mathrm{c}} \rho_{\mathrm{x}} / v_{\mathrm{x}}$. Table I also gives the maximum rate for $\mathrm{PBH}$ implosion of NSs, which we address in the next section.

TABLE I. The first five columns give the rate for compact object mergers and dark matter-induced neutron star implosions per MWEG per year $\left(A e B \equiv A \times 10^{B}\right)$, for both "Non-Implosive" and NS-imploding dark matter. ADM1 and ADM2 are defined by $t_{\mathrm{c}} \rho_{\mathrm{x}} / v_{\mathrm{x}}=3$ and $15 \mathrm{Gyr} \mathrm{GeV} / \mathrm{cm}^{3}(200 \mathrm{~km} / \mathrm{s})^{-1}$ respectively, and $\mathrm{PBH}_{\text {max }}$ is a maximally NS-imploding primordial BH model defined in Sec. IV. NS-NS, NS-BH, and BH-BH indicate standard NS and $\mathrm{BH}$ mergers, while LM-BH indicates a $\mathrm{BH}-\mathrm{BH}$ merger with at least one $\sim 1.5 M_{\odot} \mathrm{BH}$ (black merger). We use the average $\mathrm{BH}$ and NS merger rates predicted in [33]; actual merger rates may be 100 -fold larger or smaller. The final column shows the number of NS implosions expected in a $t_{\mathrm{u}} \sim$ 13 Gyr old MWEG hosting $10^{9} 1 \mathrm{~B}^{*}$-distributed NSs.

\begin{tabular}{lcccccc}
\hline \hline Model & NS-NS & NS-BH & BH-BH & LM-BH & NS Im. & Im. $/ t_{\mathrm{u}}$ \\
\hline Non-Imp. & $1 \mathrm{e}-4$ & $3 \mathrm{e}-6$ & $4 \mathrm{e}-7$ & 0 & 0 & 0 \\
ADM1 & $3 \mathrm{e}-5$ & $9 \mathrm{e}-7$ & $4 \mathrm{e}-7$ & $7 \mathrm{e}-5$ & $4 \mathrm{e}-2$ & $7 \mathrm{e} 8$ \\
ADM2 & $7 \mathrm{e}-5$ & $2 \mathrm{e}-6$ & $4 \mathrm{e}-7$ & $3 \mathrm{e}-5$ & $3 \mathrm{e}-2$ & $3 \mathrm{e} 8$ \\
PBH $_{\max }$ & $1 \mathrm{e}-4$ & $3 \mathrm{e}-6$ & $4 \mathrm{e}-7$ & $4 \mathrm{e}-11$ & $1 \mathrm{e}-7$ & 400 \\
\hline \hline
\end{tabular}




\section{RARE NEUTRON STAR IMPLOSIONS FROM PRIMORDIAL BLACK HOLES}

Black holes formed from primordial perturbations during the radiation-dominated expansion of the early universe $[42,43]$, with masses between $\sim 10^{41}-10^{50} \mathrm{GeV}$, can be captured inside and subsequently consume neutron stars $[44,45]$. As this work was being completed [46] appeared, which addresses PBH-induced NS implosions, and following [6], considers r-process elements and kilonovae produced by NS implosions. The maximum PBH-induced NS implosion rate for an MWEG found here differs markedly from [46], because we use the realistic, standard values for the NS population density, $\mathrm{PBH}$ density, and $\mathrm{PBH}$ velocity dispersion. We will find that NS implosions from primordial $\mathrm{BHs}$ (PBHs) in a typical Milky Way-like galaxy are rare.

PBHs with halo density $\rho_{\text {pbh }}$ are captured by neutron stars at a rate [44]

$$
C_{\mathrm{pbh}}=\sqrt{6 \pi} \frac{\rho_{\mathrm{pbh}}}{m_{\mathrm{pbh}}}\left(\frac{2 G M R}{v_{\mathrm{x}}}\right) \frac{1-\operatorname{Exp}\left[-\frac{3 E_{\text {loss }}}{m_{\mathrm{pbh}} v_{\mathrm{x}}^{2}}\right.}{1-\frac{2 G M}{R}},
$$

where the energy loss of a PBH transiting the NS is $E_{\text {loss }} \simeq \frac{4 G^{2} m_{\mathrm{pbh}}^{2} M}{R^{2}}\left\langle\frac{\ln \Lambda}{2 G M / R}\right\rangle$, and for a typical neutron star density profile $\left\langle\frac{\ln \Lambda}{2 G M / R}\right\rangle \sim 14.7$. With Eq. (4) it can be verified that $\mathrm{PBH}$ capture in NSs is maximized for PBH masses $m_{\mathrm{pbh}} \sim 10^{44}-10^{47} \mathrm{GeV}$. Assuming $m_{\mathrm{pbh}} \sim$ $10^{45} \mathrm{GeV}$ PBHs make up the entire dark matter density, $\rho_{\mathrm{pbh}} \simeq \rho_{\mathrm{x}}$, we find that the PBH NS implosion rate appears too low to be detectable by next generation astronomical surveys, as shown in Fig. 1 and Table I.

\section{MILKY WAY R-PROCESS ENRICHMENT AND DES BOUNDS ON QUIET KILONOVAE}

NSs imploded by dark matter may eject a substantial amount of neutrons into the interstellar medium. Ejected neutron fluid will decompress, beta decay, and form a portion of the r-process elements observed in the Milky Way $[6,31,32,47]$. R-process elements are heavy elements with atomic masses around 80,130,195, formed from neutron rich fluid at an as-yet undetermined astrophysical site. While core collapse supernovae have been historically favored as candidate sites for r-process production, recent observations of a high r-process abundance in Reticulum II, and low r-process abundance in other ultra faint dwarf galaxies, favors r-process production from rare events like a NS merger [48] or NS implosion [6]. In the case of a NS implosion, the amount of NS fluid ejected will likely depend on tidal forces during the implosion [6], which require a complete hydrodynamical simulation to be properly modelled. However, it is known that in total, $\sim 10^{4} M_{\odot}$ of r-process elements must be formed to match the abundance seen in the Milky Way [49-51]. Therefore, the amount of neutron fluid ejected per NS implosion can be bounded, by limiting the total NS mass ejected to $\sim 10^{4} M_{\odot}$ in the Milky Way. In Fig. 2, we present such

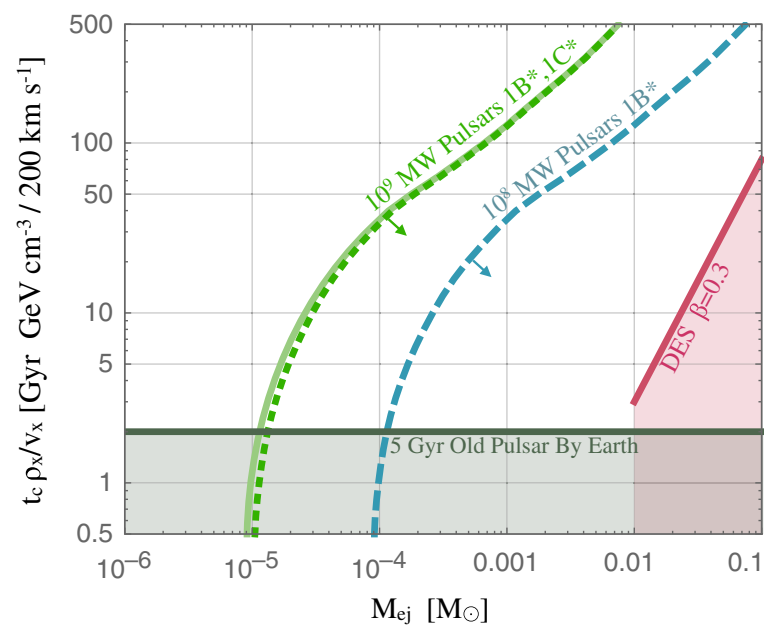

FIG. 2. Bounds on dark matter-induced quiet kilonovae as a function of the neutron fluid mass ejected $M_{\mathrm{ej}}$ during a NS implosion. The DES bounds assume kilonovae with an ejection velocity $\beta=0.3 \mathrm{c}$ [9], and assume $10^{9} \mathrm{NSs}$ in an MWEG. Milky Way $r$-process elements produced from $M_{\text {ej }}$ per NS implosion imply the indicated bounds [6], for either $10^{8}$ or $10^{9}$ total NSs, assumed to have spatial distribution $1 \mathrm{~B}^{*}$ or $1 \mathrm{C}^{*}$ given in [37] (arrows indicate the direction of excluded regions). Old pulsars in the Milky Way [1,2] also set constraints. The $\mathrm{x}$-axis indicates dark matter that implodes NSs in time $t_{\mathrm{c}}$ for background dark matter density $\rho_{\mathrm{x}}$ with velocity dispersion $v_{\mathrm{x}}$, expressed in units of $t_{\mathrm{c}} \rho_{\mathrm{x}} / v_{\mathrm{x}}$, see Eq. (2).

bounds, as a function of $t_{\mathrm{c}} \rho_{\mathrm{x}} / v_{\mathrm{x}}$. This can be compared to the final column of Table I, which shows the expected number of NS implosions after 13 Gyr.

Quiet kilonovae produced by NS-imploding dark matter can be searched for using state-of-the-art optical surveys. DES has recently published a null wide field optical search for kilonovae [9], which are the days-long luminous outbursts of beta-decaying neutron fluid ejected from NSs falling into BHs or other neutron stars. Because this search does not rely on a gravitational signature and instead seeks out beta decay emission from NS fluid flung into outer space, its findings can be used to constrain quiet kilonovae, i.e., NS fluid ejected from a NS implosion. Because kilonovae light curves depend mainly on the mass and velocity of NS fluid ejected [32], bounds obtained for NS merger kilonovae can be applied to quiet kilonovae from NS implosions. We set this bound in Fig. 2, computing the quiet kilonova rate for each $t_{\mathrm{c}} \rho_{\mathrm{x}} / v_{\mathrm{x}}$ model point, assuming an MWEG containing $10^{9} \mathrm{NSs}$.

\section{SEARCHING FOR DARK MATTER WITH NS MERGERS}

Here we show how the galactocentric radial positions of $\sim 10$ merger kilonovae would be sufficient to explore asymmetric dark matter-nucleon cross-sections orders of magnitude smaller than those presently probed using old pulsars in the Milky Way. The current generation of LV instrumentation is sensitive to gravitational strains on the 
order of $10^{-23}$ at an optimal frequency of $400 \mathrm{~Hz}$, allowing for the observation of double neutron star (NS) binaries out to distances of $\sim 70 \mathrm{Mpc}$ [8]. Anticipated upgrades will significantly expand this reach, as the amplitude of gravitational wave events is inversely proportional to the source distance, while the expected merger rate increases as the distance cubed. In the coming decade, up to hundreds of NS merger events are anticipated. Once a NS merger event is located to within $\sim 10$ square degrees by LIGO/Virgo, wide field telescopes like BlackGEM [11] and the Zwicky Transient Factory [52] are poised to image any subsequent kilonovae. The number of kilonovae found using this method will depend on their peak brightness, predictions for which range from -10 to $-16 \mathrm{AB}$ magnitude [9], while, e.g., BlackGEM will perhaps probe a $100^{\circ}$ field of view down to $-14 \mathrm{AB}$ magnitude for $200 \mathrm{Mpc}$ distant mergers. As shown in Fig. 1, galaxies with NS-imploding dark matter will have fewer NS merger kilonovae in their centers, where most NSs will have been converted to BHs. Therefore, the spatial distribution of merger kilonovae that can be used to test for dark matter. In the top panel of Fig. 3, we show the results of a cumulative distribution test, where the standard (model "1B*") NS distribution defined Sec. II, is tested against the distribution expected if dark matter is imploding neutron stars.

The altered NS merger distribution is calculated by taking the fraction of NSs converted into black holes shown in Fig. 1, and applying this conversion fraction to the $1 \mathrm{~B}^{*}$ expected distribution of NS mergers. The expected and dark matter-modified cumulative distribution functions of NS mergers in an MWEG are plotted in Appendix A. Statistical results were obtained by running 400 random Kolomogorov-Smirnov cumulative distribution trials, for each neutron star normalized implosion time $\left(t_{\mathrm{c}} \rho_{\mathrm{x}} / v_{\mathrm{x}}\right)$, to determine how many merger kilonovae located in galaxies would be necessary to detect NS-imploding dark matter at $2 \sigma$ significance. Using the same methodology, in Sec. VII we find that $\sim 20$ FRBs localized in galaxies would determine whether FRBs are a byproduct of NS implosions.

In practice, merger kilonovae occur in galaxies that are somewhat different from the Milky Way. To convert a measured galactocentric radius in a NS-merger-containing (non-Milky Way) galaxy, $r_{\mathrm{nMW}}$, to a Milky Way equivalent radius $r_{\mathrm{MW}}$, one can solve the formula

$$
\frac{\rho_{\mathrm{x}}^{\mathrm{MW}}\left(r_{\mathrm{MW}}\right)}{v_{\mathrm{x}}^{\mathrm{MW}}\left(r_{\mathrm{MW}}\right)}=\frac{\rho_{\mathrm{x}}^{\mathrm{nMW}}\left(r_{\mathrm{nMW}}\right)}{v_{\mathrm{x}}^{\mathrm{nMW}}\left(r_{\mathrm{nMW}}\right)}
$$

for $r_{\mathrm{MW}}$, where $\rho_{\mathrm{x}}$ and $v_{\mathrm{x}}$ are the dark matter density and velocity dispersion of the MWEG and non-Milky Way galaxies, as indicated. For example, the recently detected NS merger in NGC 4993 occurred in a $\sim 10^{10.9} M_{\odot}$ galaxy which would have an NFW profile defined by $\rho_{0}=$ $0.34 \mathrm{GeV} / \mathrm{cm}^{3}$ and scale factor $R_{\mathrm{s}}=7.5 \mathrm{kpc}$. The NS merger in NGC 4993 occurred at $\sim 2-3 \mathrm{kpc}$ from its center [15]. Solving Eq. (5), this corresponds to an Milky Way equivalent radius of 5-8 kpc. Note that this analysis also assumes that most identified NS mergers will have an age of $\sim 10 \mathrm{Gyr}$-indeed, the NS merger progenitor found in NGC 4993 is projected to be this old [55].
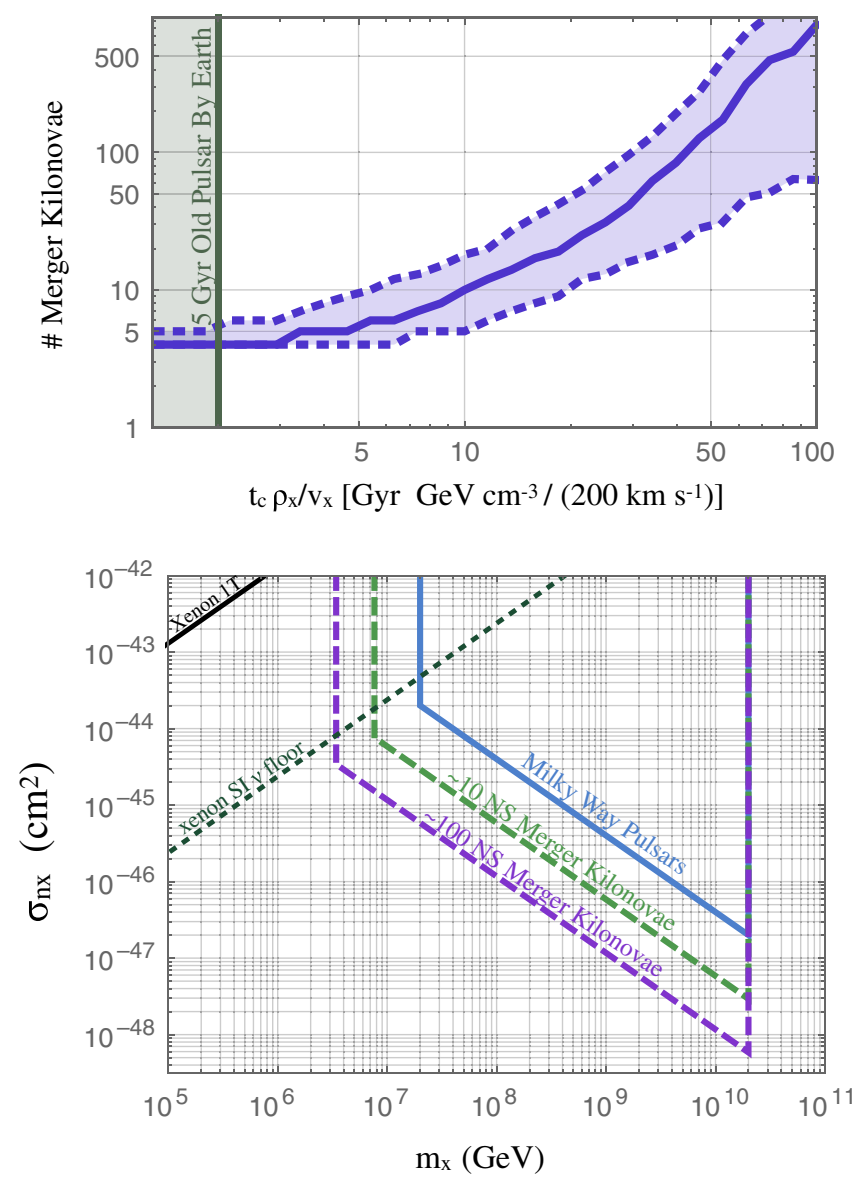

FIG. 3. Top: The number of NS mergers found by LIGO/Virgo, located to within $\sim 1 \mathrm{kpc}$ in a host galaxy by optical imaging of a kilonova, required to exclude dark matter that implodes NSs in time $t_{\mathrm{c}}$ for background dark matter density $\rho_{\mathrm{x}}$ with velocity dispersion $v_{\mathrm{x}}$, expressed in units of $t_{\mathrm{c}} \rho_{\mathrm{x}} / v_{\mathrm{x}}$, see Eq. (1) in the main text. A Kolmogorov-Smirnov test was performed at each model point against the standard hypothesis that NS mergers track a standard distribution of NSs (see Ref. [37], model 1B*) in a disc galaxy, for four hundred randomly generated merger data sets, allowing for up to $10^{3} \mathrm{NS}$ merger events per data set. Dotted bands indicate number of events needed for $2 \sigma$ significance, for the upper and lower quartile of randomly generated merger data sets; the solid line indicates the median. Bottom: The fermionic asymmetric dark matter-nucleon cross-section sensitivity obtainable with future observation of $\sim 10$ and 100 NS merger kilonovae is shown, along with the MW pulsar bound [2], Xenon $1 \mathrm{~T}$ bound [53], and the xenon neutrino floor [54], below which atmospheric neutrinos provide an irreducible background to dark matter scattering. Note that for fermionic dark matter masses $\gtrsim 10^{10} \mathrm{GeV}$, the black holes formed in NSs are too small and evaporate via Hawking radiation [19].

In Fig. 3, the per-nucleon cross-section sensitivity obtainable for heavy, asymmetric, fermionic dark matter is shown, as calculated using the capture rate and collapse conditions presented in [27,56] and Appendix B in this document. Lighter asymmetric dark matter can also be found using these methods, as in Refs. [1-5,18-24]. 

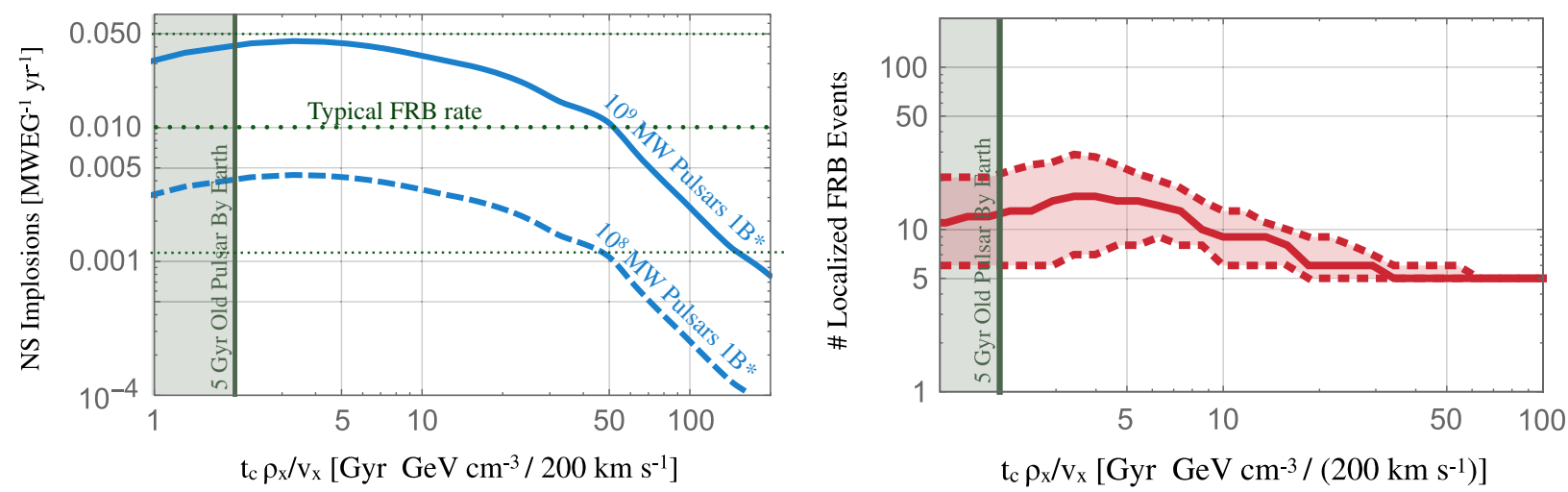

FIG. 4. Left: The NS implosion rate in a Milky Way equivalent galaxy for dark matter that implodes NSs in time $t_{\mathrm{c}}$ for background dark matter density $\rho_{\mathrm{x}}$ with velocity dispersion $v_{\mathrm{x}}$ expressed in units of $\rho_{\mathrm{x}} t_{\mathrm{c}} / v_{\mathrm{x}}$. The dotted lines indicate high, median, and low fast radio burst rate estimates from surveys [62,63]. Implosion rates are shown for $10^{8}$ and $10^{9}$ NSs with a 1B* spatial distribution [37]. Right: Number of fast radio bursts, localized to $\sim 1 \mathrm{kpc}$ in a host galaxy, required to test whether fast radio bursts originate from NSimploding dark matter, against the hypothesis that FRBs come from a nonimploding population of NSs, at $2 \sigma$ significance.

\section{FAST RADIO BURSTS FROM DARK MATTER}

Fast radio bursts (FRBs) are a newly-discovered class of millisecond-length $\sim$ Ghz radio pulses found to distances of 2 Gpc with an all sky rate $\sim 10^{4} /$ day, whose source is unknown [57,58]. In [7] it was proposed that dark matterinduced NS implosions may be the source of FRBs. The electromagnetic energy released by a NS implosion matches what is required for an FRB [59-61]. Reference [7] calculated the per MWEG NS implosion rate assuming a constant star formation history and a NS population that tracked the baryonic density in an MWEG. We improve on these rate calculations by using a realistic star formation history [34] and NS distribution [37], see Fig. 4.

The rate of NS collapse due to dark matter accumulation in the Milky Way can be estimated in several limits. In the limit of arbitrarily rapid dark matter accumulation, all NSs collapse to BHs soon after formation and the NS collapse rate is equal to the supernova rate (approximately $0.02 \mathrm{yr}^{-1}$ [64]). This scenario is ruled out by observations of Gyr old neutron stars. Intriguingly, in the case of less rapid dark matter-induced NS implosions, the overall presentday NS implosion rate actually increases due to enhanced star formation rate in the young Milky Way. While the star-formation rate of the Milky Way is currently $\sim 0.68-1.45 \mathrm{M}_{\odot} \mathrm{yr}^{-1}$ [65], the rate at redshift $z=2$ was approximately $14 \mathrm{M}_{\odot} \mathrm{yr}^{-1}$ [66-68]. If dark matter induces most 10 Gyr old NSs to implode in the present epoch, then the current implosion rate will reflect the high-redshift star formation rate. Because the dark matter density diminishes with increasing distance from the galactic center, the delay-time between NS formation and NS collapse varies considerably as a function of galactocentric radius, as evident in Fig. 1 in the main text. In Fig. 4 we plot the number of NS implosions expected per MWEG as a function of $t_{\mathrm{c}} \rho_{\mathrm{x}} / v_{\mathrm{x}}$. Upper, median, and lower FRB rates are also indicated for comparison [62,63].

\section{GRAVITATIONAL WAVES FROM A NEUTRON STAR IMPLOSION IN THE MILKY WAY}

We have identified new signatures of neutron starimploding dark matter, and fashioned qualitatively new methods for uncovering this dark sector using imminent astronomical observations. Specifically, our proposed analysis of NS merger kilonova locations has the potential to explore dark matter-nucleon scattering cross-sections up to ten orders of magnitude beyond present direct detection experiments. Finally, we note that the collapse of a neutron star into a BH could be detected directly at advanced LV, if the NS resides in the Milky Way. As we have calculated in Sec. II, NS implosion event rates may be as large as 0.05 per year. Reference [69] finds the following strain for a NS collapsing to a BH, $h_{c} \sim 5 \times 10^{-22}\left(\frac{M}{M_{\odot}}\right)\left(\frac{10 \mathrm{kpc}}{D}\right) @ 531 \mathrm{~Hz}$, so that advanced LV [70] may find an implosion out to $\sim 1 \mathrm{Mpc}$. We leave additional gravitational signatures of NS-imploding dark matter to future work, along with the application of the spatial kilonova analysis introduced here, to electromagnetic transients from exotic compact object mergers $[56,71]$.

\section{ACKNOWLEDGMENTS}

We thank Asimina Arvanitaki, Masha Baryakhtar, Shaon Ghosh, Robert Lasenby, Shirley Weishi Li, and Luis Lehner for useful discussions and correspondence. Research at Perimeter Institute is supported by the Government of Canada through Industry Canada and by the Province of Ontario through the Ministry of Economic Development \& Innovation. J. B. thanks the Aspen Center for Physics, which is supported by National Science Foundation Grant No. PHY-1066293. T. L. is partially supported by NSF Grant No. PHY-1404311 to John Beacom. 

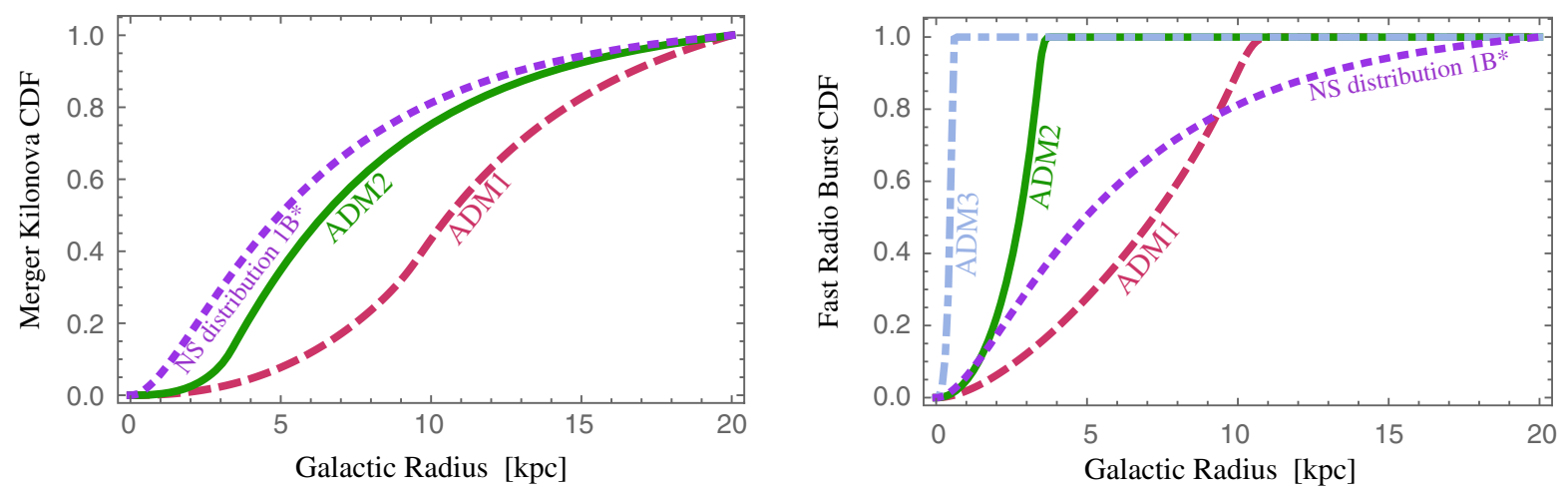

FIG. 5. Cumulative distribution functions corresponding to the Kolmogorov-Smirnov tests shown in Figs. 3 and 4 are given, for merger kilonovae (left) and fast radio bursts (right). Both merger kilonovae and FRBs are assumed to follow the distribution of NSs in an MWEG (dotted purple line labeled 1B*). This distribution can be compared with distributions for representative NS-imploding dark matter models, ADM1, ADM2, and ADM3, defined by $t_{\mathrm{c}} \rho_{\mathrm{x}} / v_{\mathrm{x}}=3,15$, and $100 \mathrm{Gyr} \mathrm{GeV} / \mathrm{cm}^{3}(200 \mathrm{~km} / \mathrm{s})^{-1}$ respectively, see Eq. (2) of the main text.

\section{APPENDIX A: CUMULATIVE DISTRIBUTION FUNCTIONS}

We include Fig. 5 that shows the cumulative distribution functions used in the Kolmogorov-Smirnov tests, whose results are displayed in Figs. 3 and 4. As indicated, the displayed CDFs here were generated using the neutron star distribution model $1 \mathrm{~B}^{*}$ in [37]. However, note that we have found that Kolmogorov-Smirnov tests utilizing other pulsar distribution models in [37] require a similar number of NS mergers to achieve sensitivity to asymmetric dark matter comparable to the results shown in Figs. 3 and 4.

\section{APPENDIX B: DARK MATTER-INDUCED NEUTRON STAR IMPLOSION TIME}

The analysis of NS implosions in Sec. I assumed that the longest timescale in the NS implosion process is the time for a NS to accrete a BH-forming mass of dark matter. Here we justify this assumption by computing timescales for all dynamical processes leading up to dark matter-induced NS implosions. As an example, we consider fermionic dark matter in the $\mathrm{PeV}$ mass range with dark matter-nucleon cross-section $\sigma_{n \mathrm{x}} \sim 10^{-45} \mathrm{~cm}^{2}$. For $\sigma_{n \mathrm{x}} \gtrsim 10^{-45} \mathrm{~cm}^{2}$, all $\mathrm{PeV}$ mass dark matter passing through the NS will be captured [56], as assumed in Sec. I. Extending this work to $\mathrm{PeV}$ mass bosonic dark matter requires minor modifications discussed around Eq. (3) of the main text, with similar results obtained. Similar results arises for low mass dark matter $[4,19,21,25,27]$, where sensitivity to $\sigma_{\mathrm{nx}}$ scales inversely with $t_{\mathrm{c}} \rho_{\mathrm{x}} / v_{\mathrm{x}}$-so that the $t_{\mathrm{c}} \rho_{\mathrm{x}} / v_{\mathrm{x}}$ reach of this study results in up to a 100-fold improvement in $\sigma_{\mathrm{nx}}$ sensitivity.

A small BH may form when a NS accumulates so much dark matter, that the dark matter cannot support its own weight with degeneracy pressure. This critical mass for fermionic dark matter is $M_{\text {crit }}^{\mathrm{f}} \sim \frac{m_{\mathrm{pl}}^{3}}{m_{\mathrm{x}}^{2}}$, as reported in the main text. In principle (e.g., for lower mass dark matter) dark matter may accumulate to $M_{\text {crit }}^{\mathrm{f}}$ size in a NS, yet not implode. This is because until dark matter in a NS "selfgravitates" or equivalently forms a bulk whose density exceeds the NS density, it will remain stable. ${ }^{2}$ We find the self-gravitating mass $M_{\mathrm{sg}}$ [19] for $\mathrm{PeV}$ mass dark matter, and determine that $M_{\text {crit }} \gg M_{\text {sg }}$, which justifies our assumption in the main text, that a $\mathrm{BH}$ will form once $M_{\text {crit }}$ dark matter accumulates. For the limiting case of a younger NS with temperature $T_{\mathrm{NS}} \sim 10^{5} \mathrm{~K}$,

$$
M_{\mathrm{sg}} \simeq 5 \times 10^{37} \mathrm{GeV}\left(\frac{T_{\mathrm{NS}}}{10^{5} \mathrm{~K}}\right)^{3 / 2}\left(\frac{\mathrm{PeV}}{m_{X}}\right)^{3 / 2}
$$

and one can see that $M_{\text {crit }}$ is at least $10^{6}$ times larger than $M_{\mathrm{sg}}$ for $\mathrm{PeV}$ mass dark matter.

Next we review the dynamical timescales for a NS to become converted to a $\mathrm{BH}$ by accumulated dark matter. First the dark matter particles thermally equilibrate with the neutron star, through repeated scattering; we denote this thermalization time scale by $\tau_{\text {th }}$. Once an unstable mass $M_{\text {crit }}$ of dark matter has thermalized into a small volume, the dark matter will collapse, cool, and form a $\mathrm{BH}$, over a time $\tau_{\mathrm{co}}$. Lastly, the small $\mathrm{BH}$ formed of dark matter accretes the surrounding neutron star in a time $\tau_{\text {Bondi }}$. We will see that each process occurs much faster than the $t_{c} \gtrsim$ Myr time scale, and conclude that the neutron star implosion time is determined by $t_{c}$.

We first consider the thermalization. Dark matter particles captured and accumulated in the neutron star thermalize with the neutrons and cool to temperature $T \simeq 10^{5} \mathrm{~K}$, same as the neutron star, before it start lose more kinetic energy and eventually collapse into a BH. The time scale $\tau_{\text {th }}$

\footnotetext{
${ }^{2}$ In the case of dark matter with substantial self-interactions, this computation is different $[2,25,26]$.
} 
is determined by the neutron-dark matter collisions [21], in particular

$\tau_{\text {th }} \simeq 8 \times 10^{-3} \mathrm{yr}\left(\frac{m_{\mathrm{x}}}{\mathrm{PeV}}\right)\left(\frac{10^{-45} \mathrm{~cm}^{2}}{\sigma_{n x}}\right)\left(\frac{10^{5} \mathrm{~K}}{T_{\mathrm{NS}}}\right)^{2}$.

After thermalization, the dark matter particles form a spherical configuration of radius $r_{0} \sim\left(9 T / 8 \pi G \rho_{c} m_{\mathrm{x}}\right)^{1 / 2}$, where the dark matter density at collapse is equal to the NS density $\rho_{c} \sim \rho_{\mathrm{n}}$. The dark matter particles can further lose more of their energy and collapse into a $\mathrm{BH}$. There are several mechanisms that contribute to this cooling, with associated time scales. Here we focus on cooling via dark matter-neutron scattering, while other cooling mechanisms can be found in, e.g., [3]. The dark matter cooling and collapse time is approximately the time for dark matter to lose $\mathcal{O}(1)$ of its kinetic energy to surrounding neutrons,

$$
\begin{aligned}
\tau_{\mathrm{co}} & \simeq \frac{1}{n \sigma_{n \mathrm{x}} v_{\mathrm{xc}}}\left(\frac{p_{F}}{\Delta p}\right)\left(\frac{m_{\mathrm{x}}}{2 m_{n}}\right) \\
& \simeq 4 \times 10^{5} \mathrm{yrs}\left(\frac{m_{\mathrm{x}}}{\mathrm{PeV}}\right)\left(\frac{10^{-45} \mathrm{~cm}^{2}}{\sigma_{\mathrm{nx}}}\right)\left(\frac{r_{x}}{r_{0}}\right),
\end{aligned}
$$

where $n$ is the number density of the neutrons. The first term $1 / n \sigma_{n \mathrm{x}} v_{\mathrm{xc}}$ is the time for a single collision. In addition, Pauli blocking has to be taken into account, as it reduces cross-section by a factor of $\Delta p / p_{F}$, hence the second term. Here $p_{F} \sim 0.5 \mathrm{GeV}$ is the neutron Fermi momentum in a NS and $\Delta p \sim m_{n} v_{\mathrm{xc}}$. The factor of $v_{\mathrm{xc}}$ here is the velocity of the dark matter sphere as it collapses through radius $r_{x}$, which can be written as $v_{\mathrm{xc}} \sim\left(G m_{\mathrm{x}} / r_{x}\right)^{1 / 2}$. The final factor takes into account that in each collision only a fraction $\sim 2 m_{n} / m_{\mathrm{x}}$ of the dark matter kinetic energy is transferred, so one requires $\sim\left(\frac{m_{\mathrm{x}}}{2 m_{n}}\right)$ collisions for an order-one energy loss.

With the $\mathrm{BH}$ formed, assuming it accretes the remainder of the NS, the time for which depends on the BHBH mass $M_{\text {crit }}$ (e.g. $\left.[6,23]\right)$.

$$
\tau_{\text {Bondi }} \sim 0.1 \operatorname{yrs}\left(\frac{m_{X}}{\mathrm{PeV}}\right)^{2}
$$

We find that $\tau_{\mathrm{th}}, \tau_{\mathrm{co}}, \tau_{\mathrm{Bondi}}$ are much shorter than $\sim t_{c}$, which therefore determines the time until a NS implodes.
[1] J. Bramante and T. Linden, Detecting Dark Matter with Imploding Pulsars in the Galactic Center, Phys. Rev. Lett. 113, 191301 (2014).

[2] J. Bramante and F. Elahi, Higgs portals to pulsar collapse, Phys. Rev. D 91, 115001 (2015).

[3] I. Goldman and S. Nussinov, Weakly interacting massive particles and neutron stars, Phys. Rev. D 40, 3221 (1989).

[4] A. de Lavallaz and M. Fairbairn, Neutron stars as dark matter probes, Phys. Rev. D 81, 123521 (2010).

[5] C. Kouvaris and P. Tinyakov, Constraining asymmetric dark matter through observations of compact stars, Phys. Rev. D 83, 083512 (2011).

[6] J. Bramante and T. Linden, On the $r$-process enrichment of dwarf spheroidal galaxies, Astrophys. J. 826, 57 (2016).

[7] J. Fuller and C. Ott, Dark matter-induced collapse of neutron stars: A possible link between fast radio bursts and the missing pulsar problem, Mon. Not. R. Astron. Soc. 450, L71 (2015).

[8] B. P. Abbott et al. (Virgo, LIGO Scientific Collaborations), Upper limits on the rates of binary neutron star and neutron star-black hole mergers from Advanced Ligo's first observing run, Astrophys. J. 832, L21 (2016).

[9] Z. Doctor et al. (DES Collaboration), A search for kilonovae in the dark energy survey, Astrophys. J. 837, 57 (2017).

[10] M. Soares-Santos et al. (DES Collaboration), A dark energy camera search for an optical counterpart to the first advanced LIGO gravitational wave event GW150914, Astrophys. J. 823, L33 (2016).

[11] S. Ghosh and G. Nelemans, Localizing gravitational wave sources with optical telescopes and combining electromagnetic and gravitational wave data, Astrophys. Space Sci. Proc. 40, 51 (2015).

[12] P. Berger et al., Holographic beam mapping of the CHIME pathfinder array, Proc. SPIE Int. Soc. Opt. Eng. 9906, 99060D (2016).

[13] L. B. Newburgh et al., HIRAX: A probe of dark energy and radio transients, Proc. SPIE Int. Soc. Opt. Eng. 9906, 99065X (2016).

[14] B. P. Abbott et al. (Virgo, LIGO Scientific Collaborations), GW170817: Observation of Gravitational Waves from a Binary Neutron Star Inspiral, Phys. Rev. Lett. 119, 161101 (2017).

[15] B.P. Abbott et al., Multi-messenger observations of a binary neutron star merger, Astrophys. J. 848, L12 (2017).

[16] K. Petraki and R. R. Volkas, Review of asymmetric dark matter, Int. J. Mod. Phys. A 28, 1330028 (2013).

[17] K. M. Zurek, Asymmetric dark matter: Theories, signatures, and constraints, Phys. Rep. 537, 91 (2014).

[18] S. D. McDermott, H.-B. Yu, and K. M. Zurek, Constraints on scalar asymmetric dark matter from black hole formation in neutron stars, Phys. Rev. D 85, 023519 (2012).

[19] J. Bramante, K. Fukushima, and J. Kumar, Constraints on bosonic dark matter from observation of old neutron stars, Phys. Rev. D 87, 055012 (2013). 
[20] N. F. Bell, A. Melatos, and K. Petraki, Realistic neutron star constraints on bosonic asymmetric dark matter, Phys. Rev. D 87, 123507 (2013).

[21] B. Bertoni, A. E. Nelson, and S. Reddy, Dark matter thermalization in neutron stars, Phys. Rev. D 88, 123505 (2013).

[22] T. Gver, A. Emre Erkoca, M. Hall Reno, and I. Sarcevic, On the capture of dark matter by neutron stars, J. Cosmol. Astropart. Phys. 05 (2014) 013.

[23] C. Kouvaris and P. Tinyakov, Growth of black holes in the interior of rotating neutron stars, Phys. Rev. D 90, 043512 (2014).

[24] Y. Kurita and H. Nakano, Gravitational waves from dark matter collapse in a star, Phys. Rev. D 93, 023508 (2016).

[25] C. Kouvaris, Limits on Self-Interacting Dark Matter, Phys. Rev. Lett. 108, 191301 (2012).

[26] J. Bramante, K. Fukushima, J. Kumar, and E. Stopnitzky, Bounds on self-interacting fermion dark matter from observations of old neutron stars, Phys. Rev. D 89, 015010 (2014).

[27] J. Bramante, Dark matter ignition of type Ia supernovae, Phys. Rev. Lett. 115, 141301 (2015).

[28] J. Bramante and J. Unwin, Superheavy thermal dark matter and primordial asymmetries, J. High Energy Phys. 02 (2017) 119.

[29] M. Baryakhtar, J. Bramante, S. Weishi Li, T. Linden, and N. Raj, Dark Kinetic Heating of Neutron Stars and An Infrared Window On WIMPs, SIMPs, and Pure Higgsinos, Phys. Rev. Lett. 119, 131801 (2017).

[30] M. Colpi, S. L. Shapiro, and I. Wasserman, Boson Stars: Gravitational Equilibria of Selfinteracting Scalar Fields, Phys. Rev. Lett. 57, 2485 (1986).

[31] J. M. Lattimer, F. Mackie, D. G. Ravenhall, and D. N. Schramm, The decompression of cold neutron star matter, Astrophys. J. 213, 225 (1977).

[32] D. Kasen, N. R. Badnell, and J. Barnes, Opacities and spectra of the $r$-process ejecta from neutron star mergers, Astrophys. J. 774, 25 (2013).

[33] J. Abadie et al. (VIRGO, LIGO Scientific Collaborations), Predictions for the rates of compact binary coalescences observable by ground-based gravitational-wave detectors, Classical Quantum Gravity 27, 173001 (2010).

[34] A. M. Hopkins and J. F. Beacom, On the normalisation of the cosmic star formation history, Astrophys. J. 651, 142 (2006).

[35] B. Paczynski, A test of the galactic origin of gamma-ray bursts, Astrophys. J. 348, 485 (1990).

[36] O. Blaes and P. Madau, Can we observe accreting, isolated neutron stars?, Astrophys. J. 403, 690 (1993).

[37] N. Sartore, E. Ripamonti, A. Treves, and R. Turolla, Galactic neutron stars. I. Space and velocity distributions in the disk and in the halo, Astron. Astrophys. 510, A23 (2010).

[38] G. Hobbs, D. R. Lorimer, A. G. Lyne, and M. Kramer, A statistical study of 233 pulsar proper motions, Mon. Not. R. Astron. Soc. 360, 974 (2005).

[39] C.-A. Faucher-Giguere and V. M. Kaspi, Birth and evolution of isolated radio pulsars, Astrophys. J. 643, 332 (2006).
[40] J. F. Navarro, C. S. Frenk, and S. D. M. White, A universal density profile from hierarchical clustering, Astrophys. J. 490, 493 (1997).

[41] Y. Sofue, Rotation curve and mass distribution in the Galactic Center-From black hole to entire galaxy, Publ. Astron. Soc. Jpn. 65, 118 (2013).

[42] S. W. Hawking, Black hole explosions, Nature (London) 248, 30 (1974).

[43] B. J. Carr and S. W. Hawking, Black holes in the early Universe, Mon. Not. R. Astron. Soc. 168, 399 (1974).

[44] F. Capela, M. Pshirkov, and P. Tinyakov, Constraints on primordial black holes as dark matter candidates from capture by neutron stars, Phys. Rev. D 87, 123524 (2013).

[45] F. Capela, M. Pshirkov, and P. Tinyakov, Constraints on primordial black holes as dark matter candidates from star formation, Phys. Rev. D 87, 023507 (2013).

[46] G. M. Fuller, A. Kusenko, and V. Takhistov, Primordial Black Holes and $r$-Process Nucleosynthesis, Phys. Rev. Lett. 119, 061101 (2017).

[47] D. Eichler, M. Livio, T. Piran, and D. N. Schramm, Nucleosynthesis, neutrino bursts and gamma-rays from coalescing neutron stars, Nature (London) 340, 126 (1989).

[48] A. P. Ji, A. Frebel, A. Chiti, and J. D. Simon, R-process enrichment from a single event in an ancient dwarf galaxy, Nature (London) 531, 610 (2016).

[49] F. van de Voort, E. Quataert, P. F. Hopkins, D. Kereš, and C.-A. Faucher-Giguère, Galactic r-process enrichment by neutron star mergers in cosmological simulations of a Milky Way-mass galaxy, Mon. Not. R. Astron. Soc. 447, 140 (2015).

[50] S. Shen, R. J. Cooke, E. Ramirez-Ruiz, P. Madau, L. Mayer, and J. Guedes, The history of R-process enrichment in the Milky Way, Astrophys. J. 807, 115 (2015).

[51] B. Wehmeyer, M. Pignatari, and F. K. Thielemann, Galactic evolution of rapid neutron capture process abundances: The inhomogeneous approach, Mon. Not. R. Astron. Soc. 452, 1970 (2015).

[52] E. Bellm, in The Third Hot-wiring the Transient Universe Workshop, edited by P. R. Wozniak, M. J. Graham, A. A. Mahabal, and R. Seaman (2014) p. 27, arXiv:1410.8185, http://www.slac.stanford.edu/econf/C131113.1/proceedings .html.

[53] E. Aprile et al. (XENON Collaboration), First Dark Matter Search Results from the XENON1T Experiment, Phys. Rev. Lett. 119, 181301 (2017).

[54] F. Ruppin, J. Billard, E. Figueroa-Feliciano, and L. Strigari, Complementarity of dark matter detectors in light of the neutrino background, Phys. Rev. D 90, 083510 (2014).

[55] P. K. Blanchard et al., The electromagnetic counterpart of the binary neutron star merger LIGO/VIRGO GW170817. VII. Properties of the host galaxy and constraints on the merger timescale, Astrophys. J. 848, L22 (2017).

[56] J. Bramante, A. Delgado, and A. Martin, Multiscatter stellar capture of dark matter, Phys. Rev. D 96, 063002 (2017).

[57] D. R. Lorimer, M. Bailes, M. A. McLaughlin, D. J. Narkevic, and F. Crawford, A bright millisecond radio burst of extragalactic origin, Science 318, 777 (2007).

[58] D. Thornton et al., A population of fast radio bursts at cosmological distances, Science 341, 53 (2013). 
[59] C. Palenzuela, Modeling magnetized neutron stars using resistive MHD, Mon. Not. R. Astron. Soc. 431, 1853 (2013).

[60] K. Dionysopoulou, D. Alic, C. Palenzuela, L. Rezzolla, and B. Giacomazzo, General-relativistic resistive magnetohydrodynamics in three dimensions: Formulation and tests, Phys. Rev. D 88, 044020 (2013).

[61] H. Falcke and L. Rezzolla, Fast radio bursts: the last sign of supramassive neutron stars, Astron. Astrophys. 562, A137 (2014).

[62] A. Rane, D. R. Lorimer, S. D. Bates, N. McMann, M. A. McLaughlin, and K. Rajwade, A search for rotating radio transients and fast radio bursts in the Parkes high-latitude pulsar survey, Mon. Not. R. Astron. Soc. 455, 2207 (2016).

[63] S. Vander Wiel, S. Burke-Spolaor, E. Lawrence, C. J. Law, and G. C. Bower, Rare event statistics applied to fast radio bursts, arXiv:1612.00896.

[64] R. Diehl et al., Radioactive Al-26 and massive stars in the galaxy, Nature (London) 439, 45 (2006).

[65] T. P. Robitaille and B. A. Whitney, The present-day star formation rate of the Milky Way determined from spitzerdetected young stellar objects, Astrophys. J. Lett. 710, L11 (2010).
[66] P. G. van Dokkum et al., The assembly of Milky Way-like Galaxies since z 2.5, Astrophys. J. 771, L35 (2013).

[67] O. N. Snaith, M. Haywood, P. Di Matteo, M. D. Lehnert, F. Combes, D. Katz, and A. Gómez, The dominant epoch of star formation in the Milky Way formed the thick disk, Astrophys. J. Lett. 781, L31 (2014).

[68] A. Marasco, V. P. Debattista, F. Fraternali, T. van der Hulst, J. Wadsley, T. Quinn, and R. Roškar, The effect of stellar feedback on a Milky Way-like galaxy and its gaseous halo, Mon. Not. R. Astron. Soc. 451, 4223 (2015).

[69] L. Baiotti, I. Hawke, and L. Rezzolla', On the gravitational radiation from the collapse of neutron stars to rotating black holes, Classical Quantum Gravity 24, S187 (2007).

[70] B. P. Abbott et al. (VIRGO, LIGO Scientific Collaborations), Prospects for observing and localizing gravitationalwave transients with Advanced LIGO and Advanced Virgo, Living Rev. Relativity 19, 1 (2016).

[71] G. F. Giudice, M. McCullough, and A. Urbano, Hunting for dark particles with gravitational waves, J. Cosmol. Astropart. Phys. 10 (2016) 001. 